\title{
Role of $5 \alpha$-reductase inhibitors in modulation of the analgesic effects of morphine: an emerging concept in pain management
}

\author{
Shailendra Kapoor
}

Received: 29 February 2012/ Accepted: 7 March 2012/Published online: 17 March 2012

(C) Springer-Verlag 2012

To the Editor,

I read with great interest the recent article by Sharif et al. [1] in a recent issue of your esteemed journal. The article is highly thought provoking. Interestingly, the past few years have seen the emergence of a number of novel new agents that alter $5 \alpha$-reductase function and thereby modulate the analgesic effects of morphine.

For instance, Moradi-Azani et al. [2] have recently demonstrated that $5 \alpha$-reductase is a modulator of formalininduced tonic pain in animal models. Central nervous system activity of $5 \alpha$-reductase is augmented by administration of morphine [3]. Not surprisingly, recent studies indicate that finasteride, as 5 alpha-reductase inhibitor, can influence and alter the perception of allodynia. Finasteride exerts its anti-nociceptive effects by augmenting the pain reducing effects of morphine and by exerting an inhibitory effect on local inflammation [4]. Finasteride also mitigates the development of opiod-induced tolerance.

Verdi and Ahmadiani [5] have recently demonstrated that naloxone-induced withdrawal symptoms can be inhibited by a single injection of finasteride. Clearly, finasteride holds significant potential in attenuating the risk of morphine dependence and decreasing the intensity of morphine withdrawal syndrome.
The above examples clearly illustrate the close relationship between finasteride, $5 \alpha$-reductase function and morphine pain modulation. Further large scale studies are needed to fully elaborate and harness the possible beneficial role of finasteride in pain modulation and morphine dependence.

\section{References}

1. Sharif A, Shoae-Hassani A, Sharif S, Banafshe HR, MortazaviTabatabaei SA, Verdi J (2012) $5 \alpha$-reductase 1 regulates spinal cord testosterone after morphine administration. Neurol Sci. doi: 10.1007/s10072-012-0936-X

2. Moradi-Azani M, Ahmadiani A, Amini H (2011) Increase in formalin-induced tonic pain by 5 alpha-reductase and aromatase inhibition in female rats. Pharmacol Biochem Behav 98:62-66

3. Amini H, Ahmadiani A (2005) In vivo evidence for an increase in 5 alpha-reductase activity in the rat central nervous system following morphine exposure. Int J Dev Neurosci 23:621-626

4. Duborija-Kovacevic N, Jakovljevic V, Sabo A, Tomic Z (2008) Anti-nociceptive and anti-inflammatory properties of 5 alphareductase inhibitor finasteride in experimental animals. Eur J Drug Metab Pharmacokinet 33:181-186

5. Verdi J, Ahmadiani A (2007) Finasteride, a 5 alpha-reductase inhibitor, potentiates antinociceptive effects of morphine, prevents the development of morphine tolerance and attenuates abstinence behavior in the rat. Horm Behav 51:605-610

S. Kapoor $(\bowtie)$

Mechanicsville, VA, USA

e-mail: shailendrakapoor@yahoo.com 